\title{
Synthèse
}

\section{Protéines de pommes de terre : vers de nouveaux axes de valorisation ?}

\author{
Virginie Deveaux-Gobert \\ Comité Nord-Plants de pommes de terre, \\ "La Pigache", \\ Avenue François-Mitterrand, \\ 62217 Beaurains \\ France \\ <gobert.virginie@free.fr>
}

\begin{abstract}
Résumé
Le tubercule de pomme de terre contient en moyenne $2 \%$ de protéines (exprimé sur poids frais) dont deux groupes ont été plus particulièrement étudiés, celui de la patatine et celui des inhibiteurs de protéases. Ces protéines solubles sont récupérées à partir des effluents de féculeries par coagulation thermique en milieu acide. De par le traitement utilisé, ces protéines ont perdu toutes leurs fonctionnalités et sont commercialisées principalement en alimentation animale. La diversité génétique présente dans ces deux familles ainsi que leurs propriétés ont longtemps été sous-estimées. La valorisation alimentaire et non alimentaire commence à se développer grâce à l'établissement de nouveaux procédés d'extraction à échelle industrielle garantissant la conservation des propriétés fonctionnelles des protéines solubles de pommes de terre. La patatine présente des propriétés émulsifiantes et moussantes tandis que les inhibiteurs de protéases peuvent être valorisés dans l'industrie pharmaceutique, dans les cosmétiques et dans la lutte contre l'obésité.
\end{abstract}

Mots clés : industrie de l'amidon ; inhibiteur de protéase ; protéine végétale ; Solanum tuberosum.

Thèmes : métabolisme ; productions végétales ; transformation ; commercialisation.

\section{Abstract \\ Potato proteins: Towards new value-added markets?}

Potato tuber contains about $2 \%$ protein (on fresh weight) dominated by two major classes, patatin and protease inhibitors. These soluble proteins are recovered from potato starch effluents by heat coagulation with an acidic $\mathrm{pH}$ adjustement. This industrial process alters their functional properties and the proteins recovered are principally used as animal food. Genetic diversity and properties of these proteins have been underestimated for a long time. New markets in human food and non-food are beginning to develop in consequence of the development of new industrial processes, which preserve the functional properties of soluble potato protein. Patatin has emulsifying and foaming properties, whereas protease inhibitors could be used in the pharmaceutical and cosmetics industries as well as in treatments against obesity.

Keywords: plant protein; protease inhibitors; Solanum tuberosum; starch industry.

Subjects: metabolism; processing; marketing; vegetal productions. es protéines solubles de pomme de terre constituent une partie importante de la charge organique des effluents de féculerie. La récupération de ces protéines à partir des "eaux de végétation " a été réalisée pour la première fois à l'échelle industrielle, en 1917 , dans une féculerie hollandaise (de Noord, 1976). À l'époque de nombreux problèmes avaient été rencontrés. La pro- duction était relativement faible et l'aspect du produit obtenu n'avait pas permis une commercialisation à un prix intéressant. C'est à partir des années 1940 que les protéines solubles de pommes de terre ont fait l'objet de nombreux et nouveaux travaux. L'objectif principal de ces derniers était de diminuer la charge polluante des effluents, tout en restant compétitif sur le marché de la fécule de 
pomme de terre. De nombreux procédés ont alors été décrits et classés dans deux grandes catégories, à savoir les méthodes de coagulation-précipitation et les méthodes de récupération des protéines ainsi précipitées : la coagulation par la chaleur (Strolle et al., 1973; de Noord, 1976), la précipitation acide (Meister et Thompson, 1976 ; Finley et Hautala, 1976 ; Knorr 1977a, 1980, 1982), la séparation par formation de mousse (Weijenberg et al., 1978), le fractionnement à sec (Holm, 1980), la complexation avec le carboxyméthylcellulose, un agent d'antiredéposition (Gonzalez et al., 1991), l'utilisation de métabisulphite de sodium (Van Driessche et al., 1982) ou de bentonite en tant qu'agent absorbant (Lindner et al., 1981), l'irradiation (Kume et Takehisa, 1984), l'osmose inverse (Rüffer et al., 1997), la centrifugation (Trump, 1988) et l'ultrafiltration (Wojnowska et al., 1981 ; Boruch et al., 1989 ; Zwijnenberg et al., 2002).

Parmi toutes ces méthodes, la coagulation par la chaleur en milieu acide est à notre connaissance, la seule qui soit utilisée à l'échelle industrielle (figure 1).

\section{Protéines solubles du tubercule}

Le tubercule de pomme de terre contient environ $2 \mathrm{~g}$ de protéines/100 $\mathrm{g}$ de matière fraîche. Lors de la campagne 2006-2007, le tonnage français de pomme de terre valorisée par l'industrie féculière s'est élevé à 1,13 Mt, soit 22600 tonnes de protéines solubles potentiellement récupérables (source GIPT, Groupement interprofessionnel pour la valorisation de la pomme de terre, http://www.gipt.net/). La valeur nutritionnelle des protéines de pomme de terre est comparable à celle des protéines de l'œuf (Van Gelder et Vonk, 1980 ; Ralet et Guéguen, 1999), faisant ainsi de ces protéines une source azotée de haute valeur, principalement en alimentation animale. Les protéines de pomme de terre sont également commercialisées comme source d'azote pour l'industrie de la fermentation. Le féculier hollandais, Avebe, a, quant à lui, obtenu l'autorisation d'utiliser les protéines de pomme de terre, ainsi que leurs hydrolysats, en alimentation humaine (Journal officiel des Communautés européennes $\mathrm{n}^{\circ} \mathrm{L} 050$ du 21/02/2002, p.0092-0093, http://eurlex.europa.eu/LexUriServ/site/fr/oj/2002/ 1_050/1_05020020221fr00920093.pdf). Il

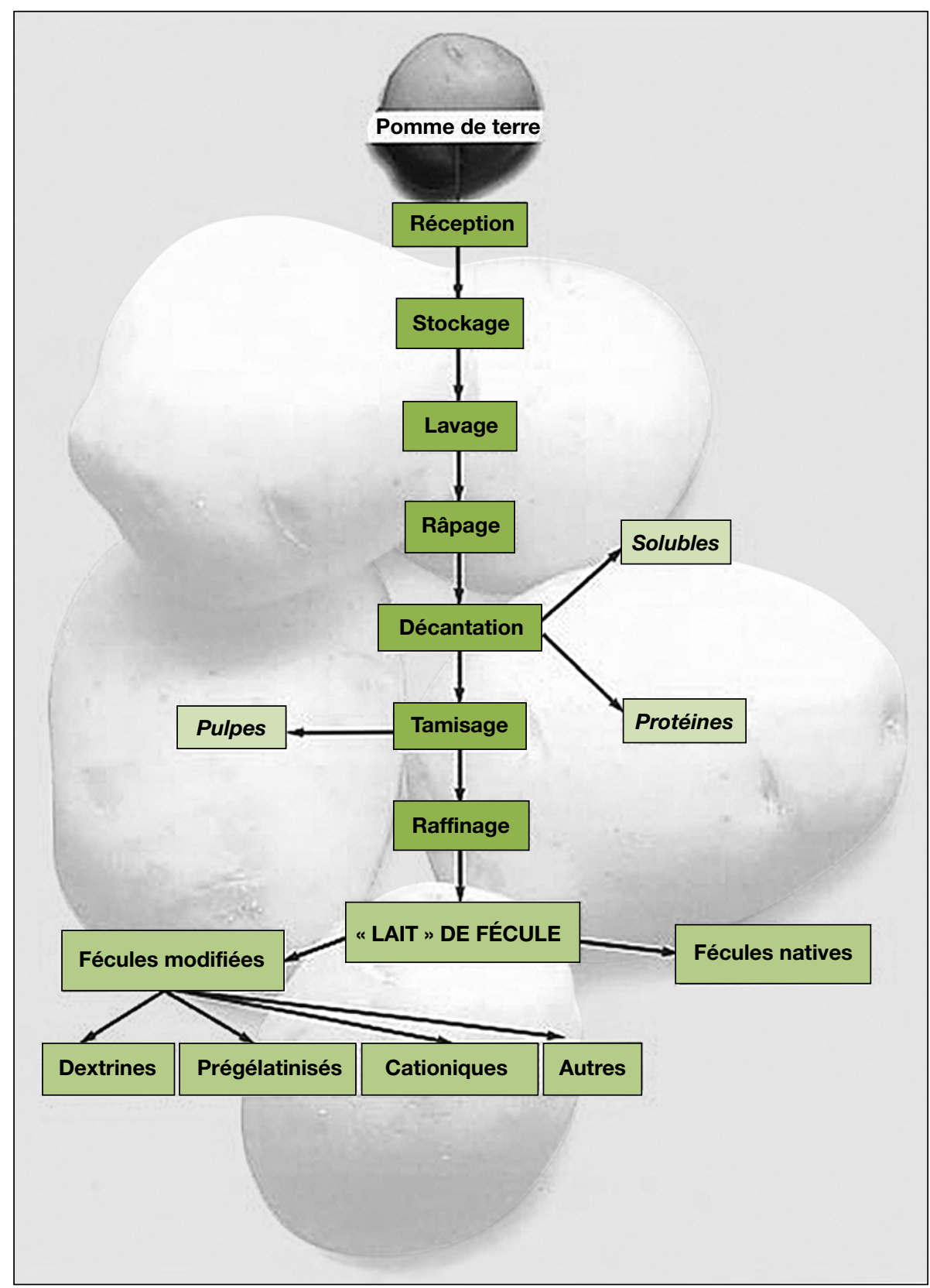

Figure 1. Procédé d'extraction de la fécule de pomme de terre ( $d$ 'après le Groupement interprofessionnel pour la valorisation de la pomme de terre, GIPT).

Figure 1. Extraction process for the potato flour according to GIPT.

Source GIPT (Groupement interprofessionnel pour la valorisation de la pomme de terre, http://www.gipt.net/)

existe différentes protéines dans le tubercule de pomme de terre, souvent réparties en trois grandes familles (figure 2); la patatine, les inhibiteurs de protéases et toutes les autres protéines présentes dans le tubercule (Pots et al., 1999b).

\section{Patatine}

La patatine (PF01734, http://pfam.sanger. ac.uk/family?acc $=$ PF01734) fait partie d'une famille multigénique de glycoprotéines de $40 \mathrm{kDa}$ (Hasson et Laties, 1976 ; Racusen et Foote, 1980 ; Paiva et al., 1983 ; Twell et Ooms, 1988).

Elle représente environ $40 \%$ des protéines totales solubles du tubercule de pomme de terre. La fonction physiologique de la patatine dans le tubercule est sujette à discussion. En effet, on lui attribue un rôle de protéine de stockage, ainsi 


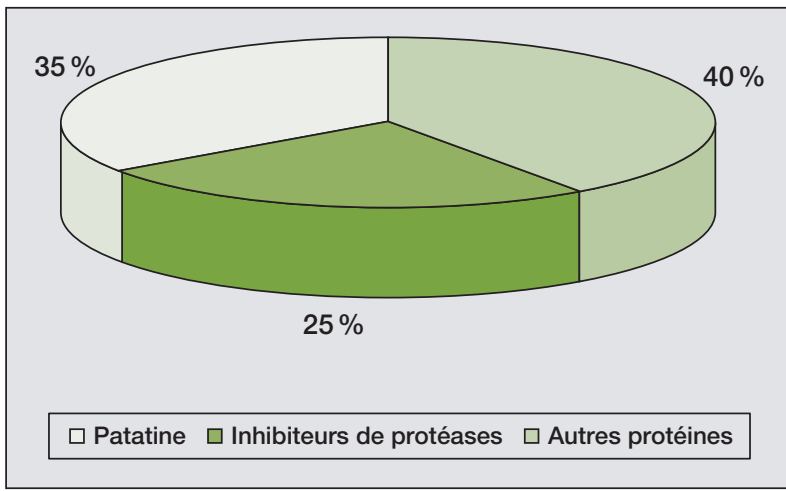

Figure 2. Proportions des protéines solubles du tubercule de pomme de terre.

Figure 2. Soluble potato tuber proteins ratios.

qu'une activité enzymatique de type lipide acyl-hydrolase et acyl-transférase (Racusen, 1983; Rosahl et al., 1987 ; Andrews et al., 1988). L'activité enzymatique de la patatine permettrait de dégrader rapidement des lipides membranaires, libérant ainsi des métabolites, nécessaires à la germination et au développement du tubercule. Il semblerait aussi que cette famille de protéines soit impliquée, dans les mécanismes de défense de la plante contre les attaques de micro-organismes et d'insectes (Strickland et al., 1995). Les acides gras ainsi libérés seraient utilisés pour la formation de cire et de certains dérivés toxiques (Racusen, 1984). Paradoxalement, les travaux de Höfgen et Willmitzer (1992), ont montré que des plantes transformées par génie génétique, présentant une réduction drastique de la quantité de patatine de $90 \%$, ne montraient aucune différence de phénotype ou de développement par rapport aux témoins. Ce résultat semble contredire les hypothèses fonctionnelles précédentes à moins de supposer que la patatine ait évolué en protéine de stockage à partir d'une protéine ancestrale ayant d'abord eu une activité métabolique (Narvaez-Vasquez et Ryan, 2002; Shewry, 2003). L'étude de la structure de la famille multigénique de la patatine (10-15 copies du gène par génome haploïde) a montré l'existence conjointe de gènes fonctionnels mais aussi de " pseudogènes ", ces derniers ayant certainement été fonctionnels avant la dégénérescence de séquence (Pikaard et al., 1986 ; Stupar et al., 2006). Les travaux de Stupar et al., 2006 ont montré que chaque copie de la patatine contribue au phénotype de la plante et subit une pression de sélection pour la fonction de protéine de stockage. Cette structure, généralement observée chez les protéines de stockage, est composée de copies fonctionnelles et de pseudogènes. Les gènes de la patatine mais aussi ceux de la zéine, protéine de stockage du maïs (Song et al., 2004), sont les cas les plus étudiés. Ces deux familles de gènes auraient ainsi évolué de la même manière. La présence de ces pseudogènes représente une source de variation allélique qui, via des recombinaisons et des événements de conversion génique, pourrait aboutir à de nouvelles copies fonctionnelles.

\section{Inhibiteurs de protéases}

La deuxième grande famille de protéines solubles du tubercule de pomme de terre représente 20 à $30 \%$ des protéines totales solubles du tubercule (Melville et Ryan, 1972). Elle est constituée d'inhibiteurs de protéases, répartis en trois classes. La première est constituée de l'inhibiteur de protéase I (PI-1, type sérine, PF00280, http:// pfam.sanger.ac.uk/family?acc $=$ PF00280)

(Melville et Ryan, 1972), la deuxième de l'inhibiteur de protéase II (PI-2, type sérine,PF02428,http://pfam.sanger.ac.uk/ family?acc=PF02428). Ces deux inhibiteurs de protéases ont la particularité d'être stables à des températures élevées (Plunkett et Ryan, 1980). La troisième classe regroupe les inhibiteurs de protéases ayant des poids moléculaires compris entre 20 et $22 \mathrm{kDa}$. Cette troisième classe est divisée en quatre groupes :

- les inhibiteurs de protéases de type Kunitz (inhibition des protéases sérines, PF00197, http://pfam.sanger.ac.uk/family? acc $=$ PF00197) ;

- les inhibiteurs de protéases de type cystéine (inhibition de la papaine (PF00112,http://pfam.sanger.ac.uk/family? acc $=$ PF00112) et de la cathepsine L) ;

- les inhibiteurs de protéases de type aspartique (inhibition de la trypsine
(PF00197,http://pfam.sanger.ac.uk/family? acc=PF00197) et de la cathepsine D) ;

- l'inhibiteur de la carboxypeptidase (PCI, PF02977, http://pfam.sanger.ac.uk/ family?acc=PF02977) présentant une activité envers la carboxypeptidase A.

Les inhibiteurs de protéases régulent et contrôlent les protéases endogènes pendant la phase de dormance du tubercule. De même que la patatine, les inhibiteurs de protéases ont à la fois un rôle de protéine de stockage et un rôle dans les mécanismes de défense (Malova et al., 1976 ; Bryant et al., 1976 ; Harsulkar et al., 1999). Ainsi, certains inhibiteurs de la troisième classe (20-22 kDa) ont la capacité d'inhiber la croissance des hyphes de Phytophthora infestans Mont de Bary (Valueva et al., 1998) et Fusarium culmorum (Speranskaya et al., 2006). La famille des inhibiteurs de protéases de la pomme de terre présente une grande diversité de séquences et d'activités antiprotéases (Pouvreau et al., 2001 ; Heibges et al., 2003), permettant ainsi à chaque variété de pomme de terre d'inhiber, potentiellement, un large spectre de pathogènes.

La patatine et les inhibiteurs de protéases constituent les protéines majoritaires solubles du tubercule de pomme de terre. La troisième famille, qui représente 20 à $30 \%$ des protéines totales solubles ayant un poids moléculaire supérieur à $40 \mathrm{kDa}$, est constituée de lectines, de polyphénoloxidases et d'enzymes impliquées dans la synthèse de l'amidon et de phosphorylases (Pouvreau, 2004).

\section{De nouveaux marchés grâce à de nouveaux procédés}

La coagulation par la chaleur, en milieu acide, a pour conséquence de dénaturer les protéines, ce qui entraîne une perte irréversible de leurs propriétés fonctionnelles. En fait, le mode de récupération influence fortement la qualité et les propriétés des protéines solubles de pomme de terre (Knorr, 1977b, 1980). Un certain nombre d'études ont donc été menées afin de déterminer la structure et les propriétés de la patatine (Pots et al., 1998a, 1998b, 1999a, 1999b, 1999c; Van Koningsveld et al., 2001) et des inhibiteurs de protéases (Pouvreau et al., 2001 ; Heibges et al., 2003; Pouvreau et al., 2003 ; Pouvreau, 2004) en relation avec la production de protéines fonctionnelles à l'échelle industrielle (Wojnowska et al., 1981; Ralet et Guéguen, 2000, 2001). C'est en accumulant les connaissances concernant les multiples fonctionnalités 
des protéines solubles de pomme de terre que de nouveaux axes de valorisation alimentaire et non alimentaire peuvent ainsi voir le jour. Par exemple, la patatine a des propriétés liantes, émulsifiantes et moussantes qui peuvent être mises à profit dans des préparations culinaires aussi diverses que des confiseries, des sauces, des entremets ou des produits de boulangerie. L'inhibiteur de protéase II, quant à lui, influence la sensation de satiété. La satiété est directement liée au taux de cholécystokinine, peptide de 33 acides aminés, sécrété lors de la digestion. Owyang et al. (1986) ont montré que les inhibiteurs de protéases de type sérine, comme l'inhibiteur de protéase II de la pomme de terre, permettaient le maintien de taux élevés en cholécystokinine, réduisant ainsi la prise alimentaire chez l'homme. La mise au point d'un procédé permettant son extraction à l'échelle industrielle tout en conservant ses propriétés a permis la commercialisation de cet inhibiteur (société Kemin ${ }^{\circledR}$ ). En termes de valorisation non alimentaire, les inhibiteurs de protéases suscitent de plus en plus d'intérêts dans l'industrie pharmaceutique et le marché des cosmétiques. En effet, les inhibiteurs de protéases du tubercule de pomme de terre et, plus particulièrement, l'inhibiteur de protéase I, l'inhibiteur de protéase II et la potato carboxypeptidase inhibitor (PCI, 39 acides aminés, Ryan et al., 1974) ont des propriétés anticancéreuses potentielles (Huang et al., 1981; Huang et al., 1997 ; Villanueva et al., 1998) et jouent aussi un rôle dans la prévention des dermatites et dans la cicatrisation des plaies. Les inhibiteurs de protéases ont également la propriété d'inhiber la croissance de diverses bactéries et champignons dans un certain nombre de systèmes in vitro. Ainsi, Kim et al., (2006) ont purifié et caractérisé un inhibiteur de protéase à sérine, appelé Potide-G $(5,5 \mathrm{kDa})$, à partir de tubercules de pommes de terre (cv. Golden Valley). Cet inhibiteur, thermostable, présente des activités antimicrobiennes sur des souches bactériennes (Staphylococcus aureus, Listeria monocytogenes, Escherichia coli, Clavibacter michiganense subsp. michiganense), mais aussi vis-àvis des champignons (Candida albicans, Rhyzoctonia solani).

\section{Création variétale}

La quantité de protéines totales et coagulables des variétés féculières de pomme de terre est vite devenue un critère de sélection variétale à la demande des industriels. En effet, des quantités élevées permettent à la fois un taux de récupération important et une réponse aux exigences environnementales. La quantité de protéines coagulables dépend de la variété (Gravoueille, 2006), et les récents travaux de Lehesranta et al., (2007) ont montré que la fertilisation avait un effet significatif sur la composition protéique du tubercule de pomme de terre. Des différences quantitatives ont été détectées à l'aide de la protéomique et concernent apparemment les protéines impliquées dans le métabolisme carboné et dans les mécanismes de défense (Lehesranta et al., 2007). Les quantités de protéines totales et coagulables font l'objet d'un programme de recherche basé sur la mise au point de marqueurs moléculaires (Comité Nord-Plants de pomme de terre, France, http://sites.terre-net.net/comite nordplant/). Par ailleurs, l'étude des proportions relatives et l'inventaire des propriétés fonctionnelles des protéines solubles de pomme de terre doivent se poursuivre puisqu'elles n'ont concerné qu'un nombre très limité de variétés, ce qui laisse à penser que ces propriétés sont pour l'instant sous-estimées et par conséquent non exploitées. Dans cette optique, le Comité Nord-Plants de pomme de terre étudie les différentes classes de protéines présentes chez les variétés et hybrides issus de la station de création variétale (Bretteville-du-GrandCaux, Haute-Normandie, France) et évalue les valorisations potentielles.

\section{Conclusion}

Les protéines solubles du tubercule de pomme de terre ont longtemps été considérées comme un coproduit des industries féculières, valorisé principalement en alimentation animale. Une meilleure connaissance de leurs structures et de leurs propriétés permet aujourd'hui l'établissement de nouveaux procédés d'extraction et donc de nouveaux axes de valorisation. La diversité génétique, d'un point de vue protéine, des variétés de pomme de terre n'est pour l'instant pas complètement exploitée. L'émergence de nouveaux marchés est donc attendue dans les années à venir.

\section{Références}

Andrews DL, Beames B, Summers MD, Park WD. Characterization of the lipid acylhydrolase activity of the major potato (Solanu$m$ tuberosum) tuber protein, patatin, by cloning and abundant expression in a baculovirus vector. Biochem J $1988 ; 252$ : 199-206.

Boruch M, Makowski J, Wachowicz M, Dubla W. Energy-saving method for obtaining protein from potato juice. Acta Aliment Hung $1989 ; X V: 253-60$.

Bryant J, Green TR, Gurusaddaiah T, Ryan CA. Proteinase inhibitor II from potatoes: isolation and characterization of its promoter components. Biochemistry-US $1976 ; 15$ : 3418-24.

de Noord KG. Production and properties of potato protein. Eurofood $1976 ; 79: 1-4$.

Finley JW, Hautala E. Recovery of soluble proteins from waste streams. Food Prod Dev $1976 ; 10: 92-3$.

Gonzalez JM, Lindamood JB, Desai N. Recovery of protein from potato plant waste effluents by complexation with carboxymethylcellulose. Food Hydrocolloid 1991; 4 : 355-63.

Gravoueille JM. Augmenter la teneur en protéines coagulables. Pomme de Terre Fr 2006 ; $547: 38-9$.

Harsulkar AM, Giri AP, Patankar AG, et al. Successive use of non-host plant proteinase inhibitors required for effective inhibition of Helicoverpa armigera gut proteinases and larval growth. Plant Physiol 1999 ; 121 : 497-506.

Hasson EP, Laties GG. Separation and characterization of potato lipid acylhydrolases. Plant Physiol 1976 ; 57 : 142-7.

Heibges A, Glaczinski H, Ballvora A, Salamini F, Gebhardt C. Structural diversity and organization of three gene families for Kunitz-type enzyme inhibitors from potato tubers (Solanu$m$ tuberosum L.). Mol Genet Genomics 2003 ; $269: 526-34$

Höfgen R, Willmitzer L. Transgenic potato plants depleted for the major tuber protein patatin via expression of antisense RNA. Plant Sci $1992 ; 87: 45-54$

Holm F. A new system for the production of starch and protein from potato. Starch-Starke $1980 ; 32: 258-62$.

Huang DY, Swanson BG, Ryan CA. Stability of proteinase inhibitors in potato tubers during cooking. J Food Sci 1981 ; 46 : 287-90.

Huang C, Ma W-Y, Ryan CA, Dong Z. Proteinase inhibitors I and II from potatoes specifically block UV-induced activator protein-1 activation through a pathway that is independent of extracellular signal-regulated kinases, c-Jun $\mathrm{N}$-terminal kinases, and P38 kinase. Proc Natl Acad Sci USA 1997 ; 94 : 11957-62.

Kim MH, Park SC, Kim JY, et al. Purification and characterization of a heat-stable serine protease inhibitor from the tubers of new potato variety "Golden Valley". Biochem Bioph Res Co 2006 ; 346 : 681-6.

Knorr D. Protein recovery from waste effluents of potato processing plants. J Food Technol $1977 ; 12: 563-80$.

Knorr D. Potato protein concentrates: the influence of various methods of recovery upon yield, compositional and functional characteristics. J Food Process Pres 1977 ; 1 : 235-47. 
Knorr D. Effect of recovery methods on yield, quality and functional properties of potato protein concentrates. J Food Sci 1980; 45 : 1183-6.

Knorr D. Effects of recovery methods on the functionality of protein concentrates from food processing wastes. J Food Process Eng $1982 ; 5: 215-30$

Kume T, Takehisa M. Effect of irradiation for recovery of organic wastes from potato starch wastewater with chitosan. Radiat Phys Chem $1984 ; 23: 579-82$

Lehesranta SJ, Koistinen KM, Massat N, et al. Effects of agricultural production systems and their components on protein profiles of potato tubers. Proteomics $2007 ; 7: 597-604$.

Lindner P, Keren R, Ben-Gera I. Precipitation of proteins from potato juice with bentonite. $J \mathrm{Sc}$ Food Agr $1981 ; 32$ : 1177-82.

Malova EL, Shul'mina Al, Mosolov VV. Study of thermostable potato proteins by the method of isoelectric focusing. Appl Biochem Micro+ 1976; 11: 516-9; (Translated from Prikladnaya Biokhimiya i Mikrobiologiya 1975 11: 576-9).

Meister E, Thompson NR. Physical-chemica methods for the recovery of protein from waste effluent of potato chip processing. J Agr Food Chem $1976 ; 24: 919-21$.

Melville JC, Ryan CA. Chymotrypsin inhibitor from potatoes: large scale preparation and characterization of its subunit components. $J$ Biol Chem $1972 ; 247$ : 3445-53.

Narvaez-Vasquez J, Ryan CA. The systemin precursor gene regulates both defensive and developmental genes in Solanum tuberosum. Proc Natl Acad Sci USA 2002 ; 99 : 15818-21.

Owyang C, Louie DS, Tatum D. Feedback regulation of pancreatic enzyme secretion: suppression of cholecystokinin release by trypsin. J Clin Invest $1986 ; 77: 2042-7$.

Paiva E, Lister RM, Park WD. Induction et accumulation of major tuber proteins of potato in stems and petioles. Plant Physiol 1983; 71 . 161-8.

Pikaard CS, Mignery GA, Ma DP, et al. Sequence of two apparent pseudogenes of the major potato tuber protein, patatin. Nucleic Acids Res potato tuber protein

Plunkett G, Ryan CA. Reduction and carboxamidomethylation of the single disulfide bond of proteinase inhibitor I from potato tubers. J Biol Chem $1980 ; 255: 2752-5$

Pots AM, de Jongh HHJ, Gruppen $\mathrm{H}$, et al. Heat-induced conformational changes of patatin, the major potato tuber protein. Eur $J$ Biochem 1998a ; $252: 66-72$

Pots AM, De Jongh HHJ, Gruppen H, et al. The $\mathrm{pH}$ dependence of the structural stability of patatin. J Agr Food Chem 1998b ; $46: 2546-53$
Pots AM, ten Grotenhuis E, Gruppen $\mathrm{H}$, et al. Thermal aggregation of patatin studied in situ. J Agr Food Chem 1999a ; 47 : 4600-5.

Pots AM, Gruppen H, Van Diepenbeek R, et al. The effect of storage of whole potatoes of three cultivars on the patatin and protease inhibitor content; a study using capillary electrophoresis and MALDI-TOF mass spectrometry. J Sci Food Agr 1999b ; 79 : 1557-64.

Pots AM, Gruppen $\mathrm{H}$, Hessing $M$, et al. Isola tion and characterization of patatin isoforms. J Agr Food Chem 1999c ; 47 : 4587-92.

Pouvreau L, Gruppen H, Piersma SR, et al. Relative abundance and inhibitory distribution of protease inhibitors in potato juice from cv. Elkana J Agr Food Chem 2001 ; 49 : 2864-74.

Pouvreau L, Gruppen H, van Koningsveld GA et al. The most abundant protease inhibitor in potato tuber (cv. Elkana) is a serine protease inhibitor from the Kunitz family. J Agr Food Chem $2003 ; 51$ : 5001-5.

Pouvreau L. Occurrence and physico-chemical properties of protease inhibitors from potato tuber (Solanum tuberosum). Thèse de doctorat, université de Wageningen, Pays-Bas, 2004 http://library.wur.nl/wda/dissertations/dis3602.pdf.

Racusen D, Foote M. A major soluble glyco protein of potato tubers. J Food Biochem $1980 ; 4: 43-52$.

Racusen D. Occurrence of patatin during growth and storage of potato tubers. Can J BOt $1983 ; 61: 370-3$

Racusen D. Lipid acyl hydrolase of patatin. Can J Bot $1984 ; 62$ : 1640-4.

Ralet M-C, Guéguen J. Les protéines de pomme de terre: composition, isolement et propriétés fonctionnelles. Sci Aliment 1999 $19: 147-65$.

Ralet M-C, Guéguen J. Fractionation of potato proteins: solubility, thermal coagulation and emulsifying properties. Lebensm Wiss Tech nol $2000 ; 33: 380-7$.

Ralet M-C, Guéguen J. Foaming properties of potato raw proteins and isolated fractions. Lebensm Wiss Technol 2001 ; 34 : 266-9.

Rosahl S, Schell J, Willmitzer L. Expression of a tuber-specific storage protein in transgenic tobacco plants: demonstration of an esterase activity. EMBO J $1987 ; 6$ : 1155-9.

Rüffer H, Kremser U, Seekamp M. Experiences with a reverse osmosis pilot plant for the concentration of potato fruit water in the potato starch industry. Starch-Starke 1997 $49: 354-9$.

Ryan CA, Hass GM, Kuhn RW. Purification and properties of a carboxypeptidase inhibitor from potatoes. J Biol Chem $1974 ; 249$ : 5495-9.

Shewry PR. Tuber storage proteins. Ann BotLondon 2003 ; 91 : 755-69.
Song R, Segal G, Messing J. Expression of the sorghum 10-member kafirin gene cluster in maize endosperm. Nucleic. Acids Res. 2004 http://nar.oxfordjournals.org/cgi/reprint/32/22/ e189.

Speranskaya AS, Krinitsina AA, Revina TA, et al. Heterologous expression, purification, and properties of a potato protein inhibitor of serine proteinases. Biochemistry-Moscowt serine proteinases. Biochemistry-Moscow+ 2006 ; 71: 1176-82; (Translated
Biochemistry-USSR 2006;71:1451-8).

Strickland JA, Orr GL, Walsh TA. Inhibition of Diabrotica larval growth by patatin, the lipid acyl hydrolase from potato tubers. Plant Physiol $1995 ; 109: 667-74$.

Strolle EO, Cording J, Aceto NC. Recovering potato proteins coagulated by steam injection heating. J Agric Food Chem $1973 ; 21$ : 974-7.

Stupar RM, Beaubien KA, Jin W, et al. Structural diversity and differential transcription of the patatin multicopy gene family during potato tuber development. Genetics $2006^{\circ}$. $172: 1263-75$

Trump CE. By-product recovery from a potato waste plant by centrifugation. 1988 Food Processing Waste Symposium. Atlanta: Georgia Institute of Technology, 1988.

Twell D, Ooms G. Structural diversity of the patatin gene family in potato cv. Desirée. Mol Gen Genet $1988 ; 212$ : 325-36.

Valueva TA, Revina TA, Kladnitskaya GV, Mosolov VV. Kunitz-type proteinase inhibitors from intact and Phytophthora-infected potato tubers. FEBS Lett 1998 ; 426 : 131-4.

Van Driessche E, Beeckmans S, Dejaegere R, Kanarek L. On the use of sodium metabisulphite during the extraction of proteins from phenol-rich tissues. Arch Int Phys Bioch 1982 ; 90 : B222-B223.

Van Gelder WMJ, Vonk CR. Amino acid composition of coagulable protein from tubers of 34 potato varieties and its relationship with protein content. Potato Res $1980 ; 23$ : 427-34.

Van Koningsveld GA, Gruppen $\mathrm{H}$, de Jon$\mathrm{gh} \mathrm{HHJ}$, et al. Effects of $\mathrm{pH}$ and heat treatments on the structure and solubility of potato proteins in different preparations. J Agric Food Chem $2001 ; 49: 4889-97$.

Villanueva J, Canals F, Prat S, et al. Characterization of the wound-induced metallocarboxypeptidase inhibitor from potato. FEBS Lett $1998 ; 440: 175-82$

Weijenberg DC, Mulder JJ, Drinkenburg AAH, Stemerding S. The recovery of protein from potato juice wastewater by foam separation. Ind Eng Chem Proc DD 1978 ; 17 : 209-13.

Wojnowska I, Poznanski S, Bednarski W. Processing of potato concentrates and their properties. J Food Sci 1981; 47 : 167-72.

Zwijnenberg HJ, Kemperman AJB, Boerrigter $\mathrm{ME}$, et al. Native protein recovery from potato fruit juice by ultrafiltration. Desalination $2002 ; 144: 331-4$. 